\title{
Nomogram for predicting severe morbidity after pheochromocytoma surgery
}

\author{
Hongyan Wang, Bin Wu, Zichuan Yao, Xianqing Zhu, Yunzhong Jiang and Song Bai \\ Department of Urology, Shengjing Hospital of China Medical University, Shenyang, China
}

Correspondence should be addressed to S Bai: baisongcmu81@163.com

\begin{abstract}
Purpose: Although resection is the primary treatment strategy for pheochromocytoma, surgery is associated with a high risk of morbidity. At present, there is no nomogram for prediction of severe morbidity after pheochromocytoma surgery, thus the aim of the present study was to develop and validate a nomogram for prediction of severe morbidity after pheochromocytoma surgery.

Methods: The development cohort consisted of 262 patients who underwent unilateral laparoscopic or open pheochromocytoma surgery at our center between 1 January 2007 and 31 December 2016. The patients' clinicopathological characters were recorded. The least absolute shrinkage and selection operator (LASSO) binary logistic regression model was used for data dimension reduction and feature selection, then multivariable logistic regression analysis was used to develop the predictive model. An independent validation cohort consisted of 128 consecutive patients from 1 January 2017 and

31 December 2018. The performance of the predictive model was assessed in regards to discrimination, calibration, and clinical usefulness.

Results: Predictors of this model included sex, BMI, coronary heart disease, arrhythmia, tumor size, intraoperative hemodynamic instability, and surgical duration. For the validation cohort, the model showed good discrimination with an AUROC of 0.818 $(95 \% \mathrm{Cl}, 0.745,0.891)$ and good calibration (Unreliability test, $P=0.440)$. Decision curve analysis demonstrated that the model was also clinically useful.

Conclusions: A nomogram was developed to facilitate the individualized prediction of severe morbidity after pheochromocytoma surgery and may help to improve the perioperative strategy and treatment outcome.
\end{abstract}

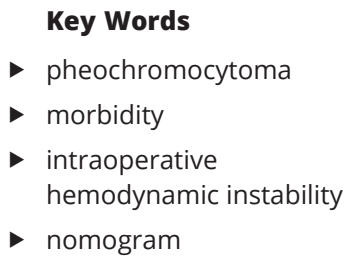

Endocrine Connections (2020) 9, 309-317

\section{Introduction}

A pheochromocytoma is a rare neuro-endocrine tumor that arises from the chromaffin cells of the adrenal medulla with an incidence of $0.2-0.8$ cases/100,000 persons/year, which present in $0.1-1 \%$ of patients with hypertension and approximately $5 \%$ of those with adrenal incidentaloma $(1,2)$. Pheochromocytomas include a series of clinical symptoms due to excessive catecholamine production, including hypertension, headache, excessive perspiration, palpitations, tremors, and facial pallor. These symptoms are often paroxysmal and can be spontaneous or induced by a variety of events, such as strenuous physical exertion, delivery, trauma, anesthesia induction, and surgery (3).

Although the mainstay strategy for treatment of pheochromocytomas, surgery is associated with a high risk of perioperative morbidity (4). It has been reported that mortality can be as high as $50 \%$. However, due to widespread improvements in preoperative medical preparation, anesthesia, and surgical techniques, the mortality rate has been significantly reduced to $0-2.9 \%$ (3). Nonetheless, morbidity is still common and difficult https://ec.bioscientifica.com

https://doi.org/10.1530/EC-20-0004 (c) 2020 The authors Published by Bioscientifica Ltd

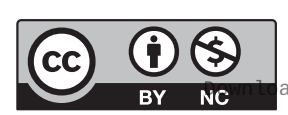

This work is licensed under a Creative Commons Attribution-NonCommercial 4.0 International License. ded from Bioscientifica.com at 04/26/2023 10:19:40AM 
to manage (5). Our previous study also demonstrated that the incidence of severe morbidity after surgery was as high as $29.8 \%$ (6). At present, risk factors related to surgeryassociated morbidity remain unclear due to the limited number of studies about this issue and the inconsistency of the conclusions.

A nomogram derived from predictive model is accepted as a reliable tool for predicting risk by incorporating and illustrating important predictors of significant clinical outcomes (7) and assigning a numerical probability to the event. Therefore, the aim of the present study was to develop and validate a nomogram for prediction of severe morbidity after pheochromocytoma surgery.

\section{Methods}

\section{Patients}

Ethical approval (Ethical Committee No. 2019PS003K) was provided by the Institutional Research and Ethics Committee of the Shengjing Hospital Affiliated China Medical University in Shengyang, China, 14 January 2019. Informed consent from all eligible patients was obtained. The clinical research registry UIN is ChiCTR1900020811.

The development cohort of this study consisted of 262 patients who underwent pheochromocytoma adrenalectomy at our center between 1 January 2007 and 31 December 2016, while the validation cohort consisted of 128 consecutive patients from 1 January 2017 and 31 December 2018 who met the same inclusion and exclusion criteria. A flowchart illustrating the patient selection process is detailed in Supplementary Fig. 1 (see section on supplementary materials given at the end of this article).

A diagnosis of pheochromocytoma was confirmed by pathological examination and patients who underwent either unilateral laparoscopic or open adrenalectomy were included. The clinical stage was localized (apparently benign) with an American Society of Anesthesiologists (ASA) score of 1-3. Patients with a familial history of pheochromocytoma, those who were converted to laparotomy or underwent bilateral adrenalectomy or surgery for an ectopic pheochromocytoma were excluded.

\section{Baseline characteristics}

Patient demographics (sex, age, BMI), comorbidity (ASA score, history of diabetes mellitus, coronary heart disease (CHD), hypertension, arrhythmia), disease characteristics (tumor side and size, tumor necrosis, enhanced CT difference), and extensive preoperative (use of alpha adrenoreceptor antagonists or crystal/colloid fluids, preoperative transfusion, 24-hour urine metanephrines/ normal upper limit), intraoperative (surgical approach, surgical duration, intraoperative hemodynamic instability (IHD), estimated blood loss), and postoperative (severe morbidity) data were recorded.

\section{Outcomes}

Data on severe morbidity were collected and classified according to the Clavien-Dindo guidelines (8), with grade II and above indicating severe morbidity and grade I indicating mild morbidity during hospitalization. IHD was defined as the presence of at least once instance of intraoperative systolic blood pressure (SBP) greater than $200 \mathrm{mmHg}$ and a mean arterial pressure (MAP) less than $60 \mathrm{mmHg}$, or the requirement for norepinephrine management or blood transfusion to maintain normal blood pressure intraoperatively (9).

Continuous invasive arterial blood pressure monitoring and vasoactive agents administration are necessary to avoid IHD, such as sodium nitroprusside for controlling hypertension and norepinephrine or blood transfusion for hypotension.

Patients with typical biochemical and radiographic presentations of pheochromocytoma were treated with doxazosin, terazosin, or prazosin for at least 1-2 weeks before surgery. A beta adrenergic blocker was added to control for tachycardia, if necessary, but only added after alpha-blockade was applied. Fluid intake was encouraged. Patients with hypertension or a larger tumor size were treated by i.v. crystalloid and colloid fluid $(2000 \mathrm{~mL} /$ day $)$ or blood transfusion at 2-3 days before surgery. The criteria for preoperative medical preparation efficacy included blood pressure less than 130/80 $\mathrm{mmHg}$, heart rate less than 90 beats/min, and hematocrit less than 0.45 .

\section{Statistical analysis}

Data were analyzed using IBM SPSS Statistics for Windows, version 22.0. (IBM Corporation), STATA 15.0. (Stata Corp., College Station, TX, USA), and R software (version 3.0.1; https://www.r-project.org/). The 'rms' and 'glmnet' packages in $\mathrm{R}$ were used in this study. The reported statistical significance levels were all two-sided, with a probability $(P)$ value of less than 0.05 considered statistically significant.

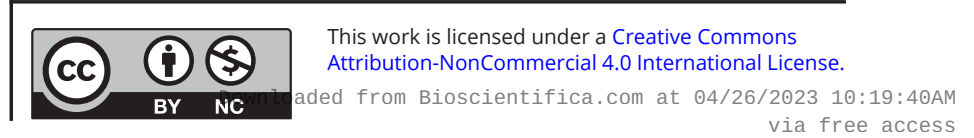




\section{Univariate analyses}

Normality of continuous variables was determined using the Kolmogorov-Smirnov test. Normally distributed continuous variables were presented as the mean \pm s.D., while non-normally continuous variables are presented as the median (interquartile range). The means of two continuous normally distributed variables were compared using the independent samples Student's $t$-test. The Mann-Whitney U test was used to compare two continuous non-normally distributed variables. Categorical variables are reported as the number (percentage). The chi-squared and Fisher's exact tests were used for comparison of categorical variables. In addition, patients with any missing data of the eligible variables were excluded from subsequent analysis.

The least absolute shrinkage and selection operator (LASSO) method, which is suitable for the reduction of high-dimensional data, was used to select the most useful predictive features from the primary data set in this study. All the clinicopathologic variables were reduced to limited potential predictors on the basis of 262 patients in the development cohort using the LASSO binary logistic regression model. If the penalization coefficient lambda $(\lambda)$ is large, there is no effect on the estimated regression parameters, but as the $\lambda$ gets smaller, some coefficients may be shrunk toward zero. We then selected the optimal $\lambda$ in the LASSO model by using ten-fold cross-validation via minimum criteria and one s.E. of the minimum criteria (the 1-s.e. criterion). Finally, the model was re-fit by using all of the non-zero coefficients, which were selected by Lasso method.

The performance of this model was tested in the independent validation cohort. The logistic regression formula formed in the development cohort was applied to the validation cohort and used to calculate the probability for each patient. To quantify the discrimination performance of the model, the area under the receiver operating characteristic (AUROC) curve was measured. An AUROC of 0.5 indicated no discrimination, whereas 1.0 indicates perfect discrimination. Calibration plots were used to assess the calibration of the model, accompanied with both the unreliability test and the HosmerLemeshow $(\mathrm{H}-\mathrm{L})$ chi-square statistic $(P>0.05$ indicates good calibration). Perfect calibration was indicated by a slope on the $45^{\circ}$ line. Decision curve analysis was conducted to determine the clinical usefulness of the model by quantifying the net benefits at different threshold probabilities in the validation cohort.

\section{Results}

After carefully screening with the same inclusion and exclusion criteria, 262 patients were included in the development cohort and 128 in the validation cohort. Of these, $78(29.8 \%)$ patients in the development cohort and $43(33.6 \%)$ in the validation cohort had severe morbidity (Clavien grade II and above), see details in Table 1.

In the univariate analysis of development cohort, sex, BMI, CHD, arrhythmia, tumor size, tumor necrosis, IHD, and surgical duration were significantly associated with severe morbidity (Tables 1 and 2). Because the sample size in this study was inadequate to satisfy the recommended guide of events per variable (10), LASSO binary logistic regression was used to construct the model. Based on all the relevant variables, The $\lambda$ value was 0.017 . Twentyone features were reduced to seven potential predictors on the basis of the development cohort (Fig. 1). The seven variables with nonzero coefficients in the LASSO logistic regression model (i.e. sex, BMI, CHD, arrhythmia, tumor size, IHD, and surgical duration) were employed in the final model. Based on these results, we developed a prediction model and then a nomogram to predict the risk of severe morbidity after pheochromocytoma surgery (Fig. 2 and Table 3).

Each clinicopathological feature corresponded to a specific point by drawing a line straight upward to the points axis. After the sum of the points was located on the total points axis, the sum representing the probability of severe morbidity was determined by drawing a line straight down to the risk axis. For example, a female patient (13 points) with history of CHD (12 points) and arrhythmia (19 points), her BMI was $20.0 \mathrm{~kg} / \mathrm{m}^{2}$ (71 points), tumor size was $8 \mathrm{~cm}$ (14 points), surgical duration was $150 \mathrm{~min}$ (11 points), and IHD (15 points) occurred during surgery. This patient was assigned a score of 155 points and the suspected probability of postoperative severe morbidity was approximately 90\% (Supplementary Fig. 2). This calculated outcome could be used in decision making for treatment plans.

The AUROC values of the development and validation cohorts were 0.807 and 0.818 , respectively, and the cutoff value of risk probability in this model was $29.5 \%$ with a sensitivity of $76.9 \%$ and specificity of $73.9 \%$ (Fig. 3A, B and Table 2). The unreliability test statistic for calibration in validation was 0.01 with a $P$ value of 0.440 and the $\mathrm{E}_{\max }$ and $\mathrm{E}_{\mathrm{avg}}$ values of 0.084 and 0.049 , respectively (Fig. 3C). The H-L chi-square statistic was 10.01 with a $P$ value of 0.4396 , which suggested good calibration. https://ec.bioscientifica.com

https://doi.org/10.1530/EC-20-0004 (c) 2020 The authors Published by Bioscientifica Ltd

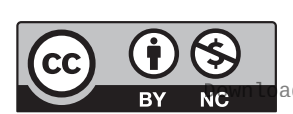

This work is licensed under a Creative Commons Attribution-NonCommercial 4.0 International License. ded from Bioscientifica.com at 04/26/2023 10:19:40AM via free access 


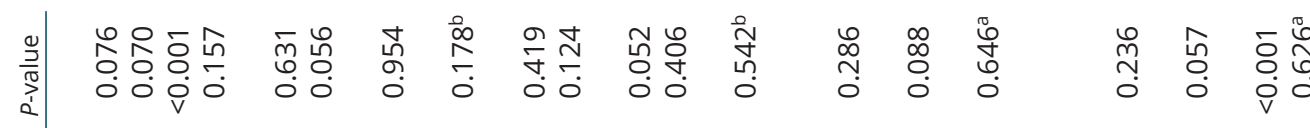

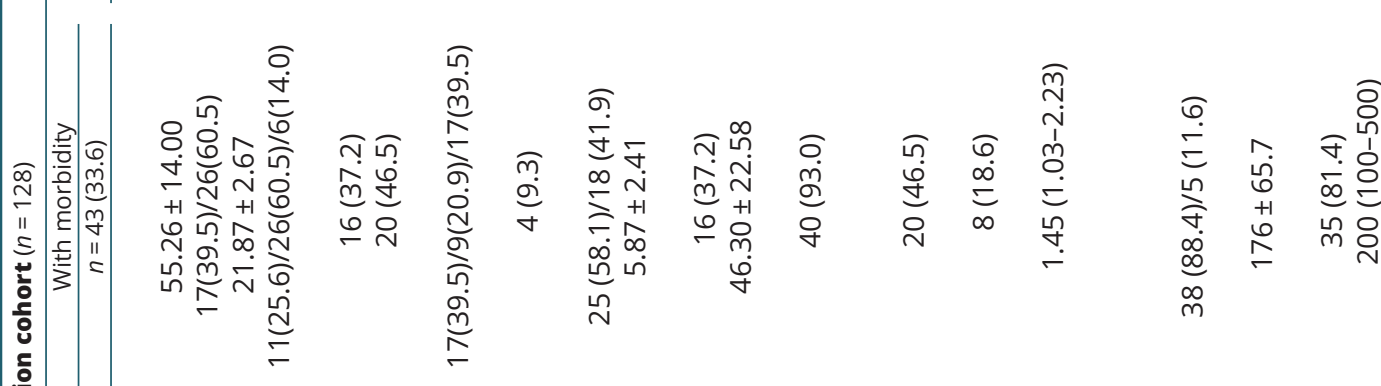

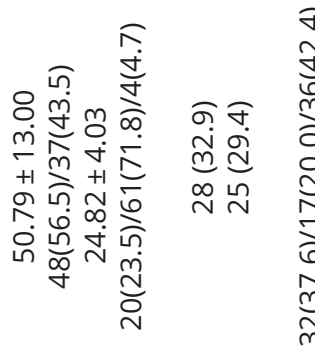

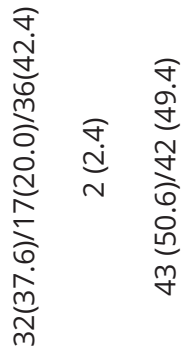

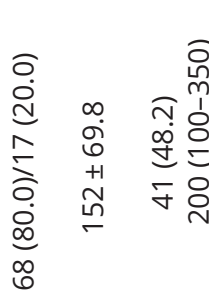

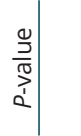

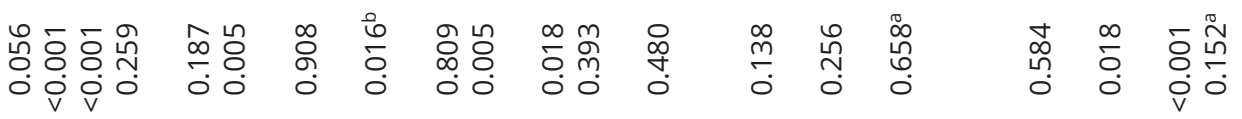

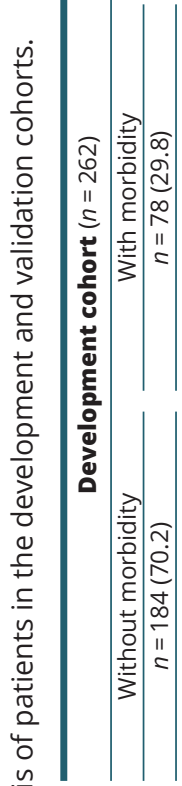

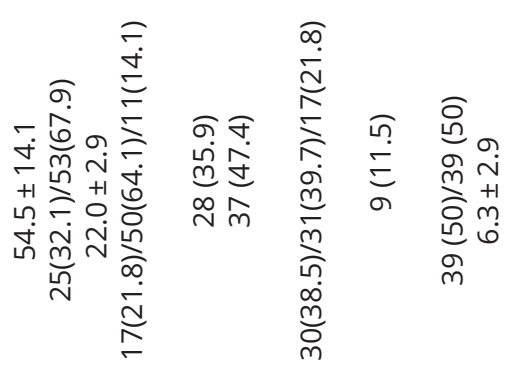

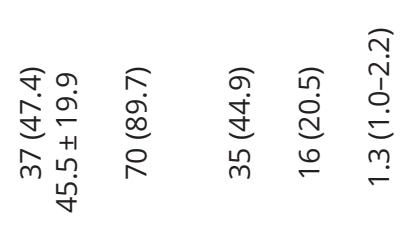

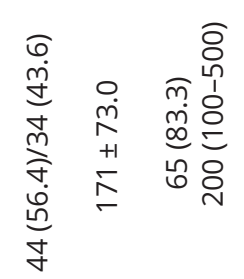

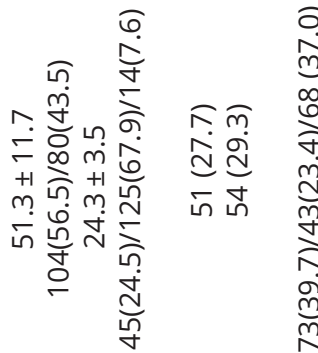

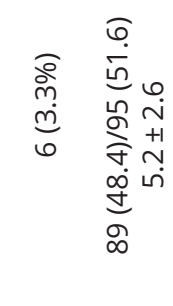

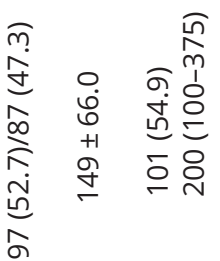

$>$

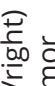

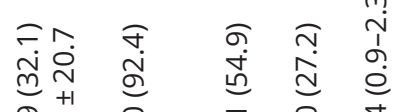

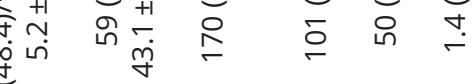

(c) 2020 The authors

Published by Bioscientifica Ltd 
Table 2 Severe morbidity of pheochromocytoma surgery.

\begin{tabular}{l}
\hline Complications \\
\hline Severe morbidity \\
Clavien II \\
Postoperative prolonged hypotension \\
Blood transfusion \\
Delirium \\
Arrhythmia \\
Pneumonia \\
Clavien III \\
Hydrothorax (thoracocentesis) \\
Hemorrhage (open laparotomy) \\
Clavien IV \\
Myocardial infarction \\
Respiratory function failure \\
Pulmonary embolism/deep vein thrombosis \\
Stroke \\
Ventricular fibrillation/flutter \\
Clavien IV \\
Mortality
\end{tabular}

\begin{tabular}{c}
\hline Development cohort number $262(100 \%)$ \\
\hline $78(29.8 \%)$ \\
$56(21.4 \%)$ \\
$29(11.0 \%)$ \\
$23(8.8 \%)$ \\
$1(0.4 \%)$ \\
$2(0.8 \%)$ \\
$1(0.4 \%)$ \\
$4(1.5 \%)$ \\
$3(1.1 \%)$ \\
$1(0.4 \%)$ \\
$14(5.3 \%)$ \\
$4(1.6 \%)$ \\
$6(2.1 \%)$ \\
$1(0.4 \%)$ \\
$2(0.8 \%)$ \\
$1(0.4 \%)$ \\
$4(1.5 \%)$ \\
$4(1.5 \%)$ \\
\hline
\end{tabular}

\begin{tabular}{c} 
Validation cohort number $\mathbf{1 2 8}(100 \%)$ \\
\hline $43(33.6 \%)$ \\
$39(30.4 \%)$ \\
$20(16.0 \%)$ \\
$15(11.2 \%)$ \\
$2(1.6 \%)$ \\
$1(0.8 \%)$ \\
$1(0.8 \%)$ \\
$0(0 \%)$ \\
$0(0 \%)$ \\
$0(0 \%)$ \\
$4(3.2 \%)$ \\
$0(0 \%)$ \\
$2(1.6 \%)$ \\
$1(0.8 \%)$ \\
$1(0.8 \%)$ \\
$0(0 \%)$ \\
$0(0 \%)$ \\
$0(0 \%)$
\end{tabular}

Categorical variables were reported as the number (percentage).

The decision curve showed that if the threshold probability of a patient ranged from $15 \%$ to $65 \%$, the use of this nomogram to predict severe morbidity after pheochromocytoma surgery was more beneficial than
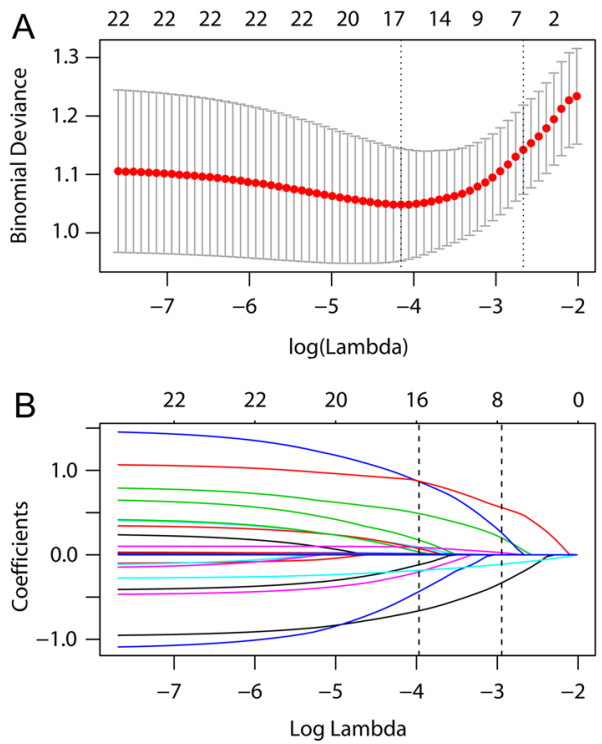

\section{Figure 1}

Texture feature selection using the least absolute shrinkage and selection operator (LASSO) binary logistic regression model. (A) The Tuning parameter $(\lambda)$ for the LASSO model was selected using ten-fold crossvalidation via minimum criteria. Dotted vertical lines were drawn at the optimal values in reference to the minimum criteria and one S.E. of the minimum criteria. (B) A coefficient profile plot was produced against the $\log (\lambda)$ sequence. A vertical line was drawn at the value selected using ten-fold cross-validation. either the treat-all-patients scheme or the treat-none scheme. Within this range, net benefit was comparable.

\section{Discussion}

Resection is the primary strategy for treatment of a pheochromocytoma, but surgery is associated with a high risk of perioperative morbidity, even though preoperative medical preparation, anesthesia, and surgical techniques have been greatly improved in recent years (6). Exploring the risk predictors for morbidity after pheochromocyma surgery will lead to better treatment outcomes, specifically risk factors associated with severe morbidity. Unfortunately, few retrospective, small size studies have addressed this issue, thus the risk factors are unclear and inconsistent. Nomogram has been accepted as a reliable tool to predict risk by illustrating important predictors of clinical events. Therefore, the aim of the present study was to develop and validate a nomogram for perioperative prediction of severe morbidity after pheochromocytoma surgery.

The Clavien classification was used to evaluate complications of pheochromocytoma with a focus on severe morbidity (Clavien grade II and above). In this study, $29.8 \%$ and $33.6 \%$ of patients in the development and validation cohorts had severe morbidity, respectively. These percentages were slightly higher than those in prior reports, in which the morbidity ranged from $0 \%$ to $20 \%(11,12)$. The developed nomogram incorporated seven predictors (i.e. sex, BMI, CHD, arrhythmia,

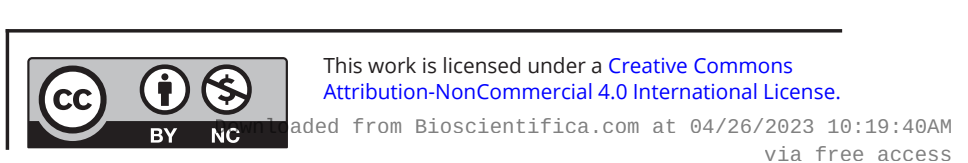




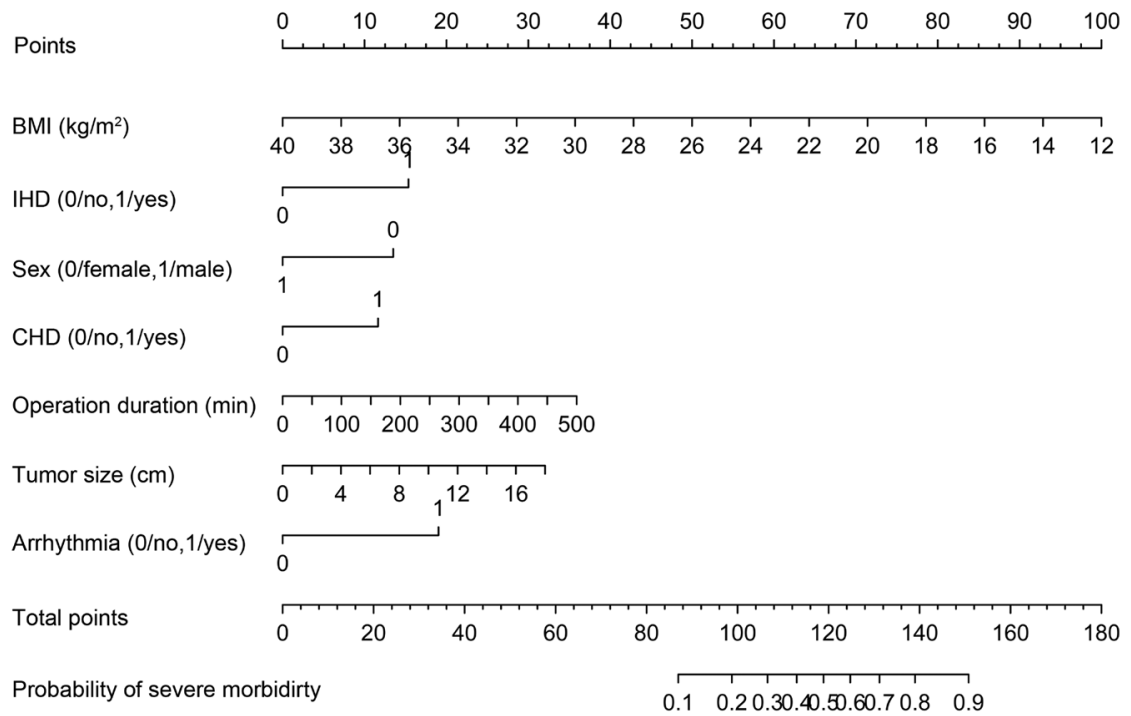

\begin{abstract}
Figure 2
Nomogram to predict severe morbidity after pheochromocytoma surgery. Each clinicopathological factor corresponds to a specific point by drawing a line straight upward to the points axis. The sum of the points located on the total points axis represents the probability of severe morbidity after pheochromocytoma surgery by drawing a line straight down to the risk axis.
\end{abstract}

tumor size, IHD, and surgical duration) based on extensive clinicopathological data to predict the risk of severe morbidity.

For the construction of this model, 21 variables were reduced to seven potential predictors based on the development cohort with the use of the LASSO binary logistic regression model. The reason that that this model was selected based on this method instead of the minimal Akaike's information criterion (AIC) was that there was less severe morbidity events compared to the number of variables, which was not in accordance with the event per variable principle. Validation of the nomogram is important to avoid overfitting and to determine generalizability. In this study, the AUROC values of the validation cohorts demonstrated adequate discrimination $(0.8183$, respectively). Calibration plots also showed optimal agreement between prediction and actual observation, as confirmed by the Unreliability test and $\mathrm{H}-\mathrm{L}$ test $(P=0.440$ and $P=0.4396$, respectively), which guaranteed the repeatability and reliability of the model. Decision curve analysis was applied in this study to explore clinical usefulness and showed that, if the threshold probability of a patient was $15 \%$ to $65 \%$, use of this nomogram to predict the probability of severe morbidity after pheochromocytoma surgery was more beneficial than either the treat-all-patients scheme or the treat-none scheme. This range also covered the cutoff value of risk probability in this model (29.5\%).

Brunaud et al. (9) demonstrated that sex (female vs male) was an independent risk predictor of morbidity after unilateral laparoscopic adrenalectomy in a retrospective study of 225 pheochromocytoma patients. In line with this, our previous study also suggested that female sex was an independent predictor of severe morbidity (odds ratio=2.62) (6). As expected, this model also included the variable of sex because females have a lower effective circulatory volume as a result of lower body weight than that of males. Moreover, these patients had peripheral

Table 3 Multivariate binary logistic regression of severe morbidity.

\begin{tabular}{l} 
Intercept and variable \\
\hline Intercept \\
Gender (female/male) \\
BMI $\left(\mathrm{kg} / \mathrm{m}^{2}\right)$ \\
Coronary heart disease \\
Arrhythmia \\
Radiographic tumor size (cm) \\
IHD \\
Duration of surgery (minutes) \\
Area under ROC curve \\
$\quad$ Development dataset \\
Validation dataset
\end{tabular}

$\begin{array}{r}\hline \multicolumn{1}{c}{\boldsymbol{\beta}} \\ \hline 2.581 \\ -0.930 \\ -0.246 \\ 0.803 \\ 1.310 \\ 0.123 \\ 1.058 \\ 0.005 \\ 0.807 \\ 0.818 \\ \hline\end{array}$

\begin{tabular}{c}
$\mathbf{9 5 \%} \mathbf{C l}$ \\
\hline$-0.032,5.193$ \\
$-1.564,0.295$ \\
$-0.358,-0.134$ \\
$0.165,1.441$ \\
$0.010,2.611$ \\
$0.006,0.239$ \\
$0.321,1.795$ \\
$0.0002,0.010$
\end{tabular}

\begin{tabular}{c} 
OR \\
\hline 13.207 \\
0.395 \\
0.782 \\
2.232 \\
3.708 \\
1.130 \\
2.880 \\
1.005
\end{tabular}

\begin{tabular}{l}
\multicolumn{1}{c}{$\mathbf{9 5 \%} \mathbf{C l}$} \\
\hline $0.969,180.053$ \\
$0.209,0.745$ \\
$0.699,0.875$ \\
$1.179,4.224$ \\
$1.010,13.615$ \\
$1.006,1.271$ \\
$1.378,6.017$ \\
$1.000,1.010$
\end{tabular}

\begin{tabular}{r}
\hline \multicolumn{1}{c}{$\boldsymbol{P}$} \\
\hline 0.053 \\
0.004 \\
$<0.001$ \\
0.014 \\
0.048 \\
0.040 \\
0.005 \\
0.039
\end{tabular}

The $\beta$ coefficient, odds ratio, and 95\% $\mathrm{Cl}$ were measured through binary logistic regression. IHD, intraoperative hemodynamic instability; OR, odds ratio.

https://ec.bioscientifica.com https://doi.org/10.1530/EC-20-0004 (c) 2020 The authors Published by Bioscientifica Ltd
$0.751,0.864$

$0.745,0.891$
0.039 

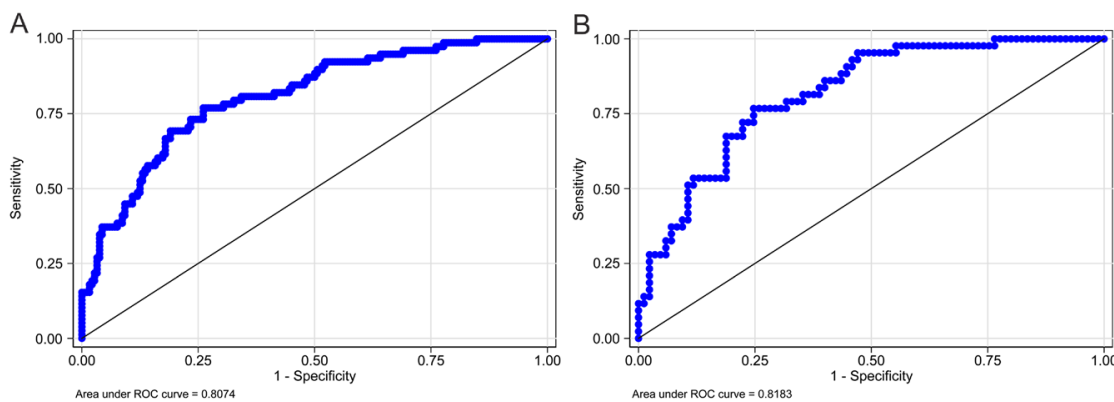

C

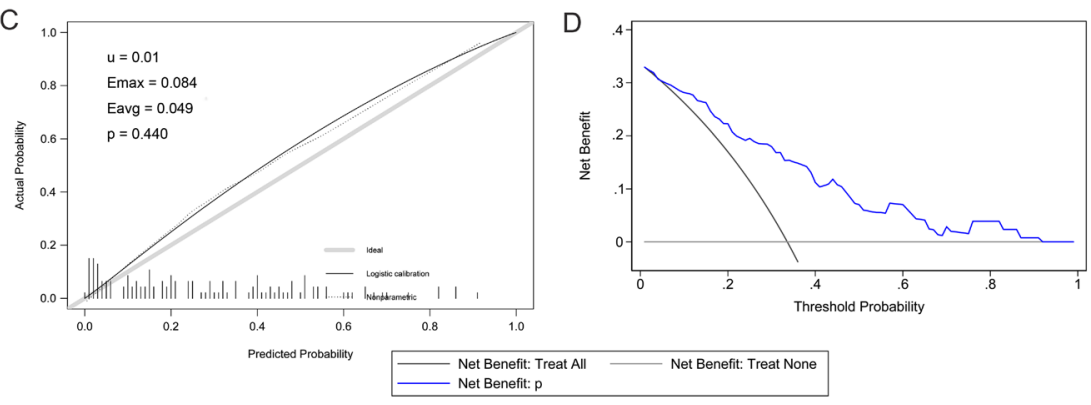

\section{Figure 3}

AUROC, calibration, and decision curve analysis for the model. (A) The AUROC of the development cohort represents the discrimination ability of the model, as measured by the C-index. (B) The AUROC of the validation cohort. (C) The calibration plot shows the relationship between the predicted probabilities based on the nomogram and the actual values of the validation cohort. A plot along the $45^{\circ}$ line indicates perfect calibration of the model, in which the predicted probabilities are identical to the actual outcomes. (D) Decision Curve Analysis: The net benefit is indicated on the $y$-axis. The blue line represents the nomogram. The black line represents the assumption that all patients have severe morbidity. The thin gray line represents the assumption that no patient has severe morbidity. vasoconstriction induced by catecholamines secreted by a pheochromocytoma, which resulted in further reduction in effective circulatory volume and large blood pressure fluctuations during the perioperative period.

The risk regarding BMI has been previously reported by our group (6), which was identified as an independent risk factor for severe morbidity. The mechanism may be the same as that of sex, as a lower BMI is associated with lower effective circulatory volume as a result of relatively lower body weight, thus resulting in large blood pressure fluctuations and a high incidence of IHD.

Usually, pheochromocytoma patients have a higher incidence of heart disease than those with essential hypertension, CHD, and arrhythmia (13). The risk predictor of this model included both CHD and arrhythmia because the myocardium and coronary arteries are exposed to abnormal elevated levels of catecholamines for prolonged periods, which could lead to collagen deposition and fibrosis in the myocardium (13). In line with this, a previous study also found that acute left cardiac dysfunction due to chronically high level epinephrine exposure was the root cause of prolonged hypotension and circulatory collapse after pheochromocytoma surgery (14).

Our study showed that tumor size was also an effective predictor of severe morbidity. A relatively larger pheochromocytoma has a more prominent network of vessels and is associated with greater blood loss during surgery than smaller tumors $(15,16)$. Meanwhile, large tumors secrete higher levels of catecholamines, which can easily lead to greater blood pressure fluctuations during the perioperative period. Natkaniec et al. (15) reported that intraoperative blood loss was significantly greater in patients with tumor diameters $\geq 6 \mathrm{~cm}$ than patients with tumor diameters $<6 \mathrm{~cm}$ based on 530 patients who underwent laparoscopic adrenalectomy. Agrusa et al. (17) and other investigators (18), however, reported that tumor size was not closely related with blood loss. One of the reasons for this discrepancy may be that the patients had various adrenal tumors in these studies, whereas our study focused on only patients with pheochromocytomas.

In this model, a longer surgical duration was also a predictor of severe morbidity. The surgical duration is prolonged due to the difficultly of the surgery, which is accompanied by greater blood loss and increased risks of organ injury and blood pressure fluctuations, subsequently resulting in postoperative morbidity. In our previous study, a longer surgical duration was also an independent risk factor for severe morbidity with an odds ratio of 2.563 (6). This finding has not previously been reported.

The definition of IHD varies; our standard was the presence of at least once instance of $\mathrm{SBP} \geq 200 \mathrm{mmHg}$ associated with a MAP $\leq 60 \mathrm{mmHg}$ or the requirement of norepinephrine agents or blood transfusion to maintain normal blood pressure intraoperatively. At present, IHD remains a common complication despite adequate preoperative medical preparation (19) and is still the most important operative and anesthetic challenge during pheochromocytoma surgery. Hypertensive episodes occur mostly during intubation and separation of the tumor, while hypotensive episodes often occur after tumor resection (20). Previous studies have reported that the incidence of IHD to be $39-48 \%$ among patients 
with pheochromocytomas (21) and was confirmed as an independent risk factor for pheochromocytoma morbidity in a previous study (9), in agreement with the present study, thus the proposed model also included IHD as a predictor of morbidity.

There were several limitations to this study that should be addressed. First, this was a retrospective study conducted in only one center. Secondly, peri-operative preparation strategies were varied and not standardized due to quite a long period of recruiting time; these differences may have influenced the final results. Thirdly, some variables that may be related to severe morbidity were not considered, such as patient symptoms, genomic characteristics, and the dose of pre-operative medical preparation, plasma metanephrine, and catecholamines in the analysis. Fourthly, this study is temporal external validation, which tested a model from older data on newer data in one center. Therefore, a multicenter cohort with standardization of pre-operative medical preparation, intra-operative anesthesia management, and the operative approach is required for validating this model. Lastly, validation using a Western cohort will also be needed prior to universal use of this nomogram, because the significant differences in genetic characteristics between the Eastern and Western cohorts should be considered. Anyhow, to the best of our knowledge, this is the first model for the prediction of severe morbidity after pheochromocytoma surgery and will likely have an impact on the treatment options, as well as the selection of patients who require additional therapy or intensive care. Doctors could perform an individualized prediction through this easyto-use scoring system.

\section{Conclusions}

A nomogram was developed to facilitate the individualized prediction of severe morbidity after pheochromocytoma surgery and may help to improve the perioperative strategy and treatment outcome.

\section{Supplementary materials}

This is linked to the online version of the paper at https://doi.org/10.1530/ EC-20-0004.

\section{Declaration of interest}

The authors declare that there is no conflict of interest that could be perceived as prejudicing the impartiality of the research reported.

\section{Funding}

This study was financially supported (MC05) through the Shengjing Hospital Science and Technology Program. Song Bai certifies that there are no conflicts of interest, including specific financial interests, relationships, and affiliations relevant to the subject matter or materials discussed in the manuscript (e.g. employment/affiliation, grants or funding, consultancies, honoraria, stock ownership or options, expert testimony, royalties, or patents filed, received, or pending).

\section{Author contribution statement}

Song Bai had full access to all the data in the study and takes responsibility for the integrity of the data and the accuracy of the data analysis. Song Bai conceptualized and designed the study. Hongyan Wang performed acquisition, analysis, and interpretation of data. Hongyan Wang and Bin Wu performed drafting of the manuscript and critical revision of the manuscript for important intellectual content. Zichuan Yao, Yunzhong Jiang, and Xianqing Zhu performed statistical analysis. Song Bai obtained funding and other (figures).

\section{Acknowledegments}

The authors give special thanks to all the teachers at the Department of Urology, Department of Graduate Medical Training, and Department of Student Affairs of Shengjing Hospital for their help and support.

\section{References}

1 Kopetschke R, Slisko M, Kilisli A, Tuschy U, Wallaschofski H, Fassnacht M, Ventz M, Beuschlein F, Reincke M, Reisch N, et al. Frequent incidental discovery of phaeochromocytoma: data from a German cohort of 201 phaeochromocytoma. European Journal of Endocrinology 2009161 355-361. (https://doi.org/10.1530/EJE-09-0384)

2 Omura M, Saito J, Yamaguchi K, Kakuta Y \& Nishikawa T. Prospective study on the prevalence of secondary hypertension among hypertensive patients visiting a general outpatient clinic in Japan Hypertension Research 200427 193-202. (https://doi.org/10.1291/ hypres.27.193)

3 Lenders JW, Eisenhofer G, Mannelli M \& Pacak K. Phaeochromocytoma. Lancet 2005366 665-675. (https://doi. org/10.1016/S0140-6736(05)67139-5)

4 Jiang M, Ding H, Liang Y, Tang J, Lin Y, Xiang K, Guo Y \& Zhang S. Preoperative risk factors for haemodynamic instability during pheochromocytoma surgery in Chinese patients. Clinical Endocrinology 201888 498-505. (https://doi.org/10.1111/cen.13544)

5 Conzo G, Pasquali D, Della Pietra C, Napolitano S, Esposito D, Iorio S, De Bellis A, Docimo G, Ferraro F, Santini L, et al. Laparoscopic adrenal surgery: ten-year experience in a single institution. $B M C$ Surgery 201313 (Supplement 2) S5. (https://doi.org/10.1186/14712482-13-S2-S5)

6 Bai S, Yao Z, Zhu X, Li Z, Jiang Y, Wang R \& Wu B. Risk factors for postoperative severe morbidity after pheochromocytoma surgery: a single center retrospective analysis of 262 patients. International Journal of Surgery 201860 188-193. (https://doi.org/10.1016/j. ijsu.2018.11.019)

7 Karakiewicz PI, Briganti A, Chun FK, Trinh QD, Perrotte P, Ficarra V, Cindolo L, De la Taille A, Tostain J, Mulders PF, et al. Multiinstitutional validation of a new renal cancer-specific survival nomogram. Journal of Clinical Oncology 200725 1316-1322. (https:// doi.org/10.1200/JCO.2006.06.1218)

8 Dindo D, Demartines N \& Clavien PA. Classification of surgical complications: a new proposal with evaluation in a cohort of 6336 https://ec.bioscientifica.com https://doi.org/10.1530/EC-20-0004 (c) 2020 The authors Published by Bioscientifica Ltd

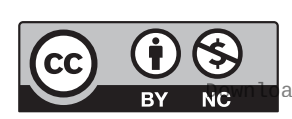

This work is licensed under a Creative Commons Attribution-NonCommercial 4.0 International License. ded from Bioscientifica com at 04/26/2023 10:19:40AM 
patients and results of a survey. Annals of Surgery 2004240 205-213. (https://doi.org/10.1097/01.sla.0000133083.54934.ae)

9 Brunaud L, Nguyen-Thi PL, Mirallie E, Raffaelli M, Vriens M, Theveniaud PE, Boutami M, Finnerty BM, Vorselaars WM, Rinkes IB, et al. Predictive factors for postoperative morbidity after laparoscopic adrenalectomy for pheochromocytoma: a multicenter retrospective analysis in 225 patients. Surgical Endoscopy 201630 1051-1059. (https://doi.org/10.1007/s00464-015-4294-7)

10 Peduzzi P, Concato J, Kemper E, Holford TR \& Feinstein AR. A simulation study of the number of events per variable in logistic regression analysis. Journal of Clinical Epidemiology 199649 1373-1379. (https://doi.org/10.1016/s0895-4356(96)00236-3)

11 Dickson PV, Alex GC, Grubbs EG, Ayala-Ramirez M, Jimenez C, Evans DB, Lee JE \& Perrier ND. Posterior retroperitoneoscopic adrenalectomy is a safe and effective alternative to transabdominal laparoscopic adrenalectomy for pheochromocytoma. Surgery 2011 150 452-458. (https://doi.org/10.1016/j.surg.2011.07.004)

12 Shen WT, Grogan R, Vriens M, Clark OH \& Duh QY. One hundred two patients with pheochromocytoma treated at a single institution since the introduction of laparoscopic adrenalectomy. Archives of Surgery 2010145 893-897. (https://doi.org/10.1001/ archsurg.2010.159)

13 Stolk RF, Bakx C, Mulder J, Timmers HJ \& Lenders JW. Is the excess cardiovascular morbidity in pheochromocytoma related to blood pressure or to catecholamines? Journal of Clinical Endocrinology and Metabolism 201398 1100-1106. (https://doi.org/10.1210/jc.2012-3669)

14 Zhang R, Gupta D \& Albert SG. Pheochromocytoma as a reversible cause of cardiomyopathy: analysis and review of the literature. International Journal of Cardiology 2017249 319-323. (https://doi. org/10.1016/j.ijcard.2017.07.014)
15 Natkaniec M, Pędziwiatr M, Wierdak M, Białas M, Major P, Matłok M, Budzyński P, Dworak J, Buziak-Bereza M \& Budzyński A. Laparoscopic adrenalectomy for pheochromocytoma is more difficult compared to other adrenal tumors. Wideochirurgia i Inne Techniki Maloinwazyjne 201510 466-471. (https://doi.org/10.5114/wiitm.2015.52869)

16 Bozkurt IH, Arslan M, Yonguc T, Degirmenci T, Koras O, Gunlusoy B \& Minareci S. Laparoscopic adrenalectomy for large adrenal masses: is it really more complicated? Kaohsiung Journal of Medical Sciences 201531 644-648. (https://doi.org/10.1016/j.kjms.2015.09.005)

17 Agrusa A, Romano G, Frazzetta G, Chianetta D, Sorce V, Di Buono G \& Gulotta G. Laparoscopic adrenalectomy for large adrenal masses: single team experience. International Journal of Surgery $2014 \mathbf{1 2}$ (Supplement 1) S72-S74. (https://doi.org/10.1016/j.ijsu.2014.05.050)

18 Carter YM, Mazeh H, Sippel RS \& Chen H. Safety and feasibility of laparoscopic resection for large $(>6 \mathrm{CM})$ pheochromocytomas without suspected malignancy. Endocrine Practice 201218 720-726. (https://doi.org/10.4158/EP12014.OR)

19 Vorselaars WMCM, Postma EL, Mirallie E, Thiery J, Lustgarten M, Pasternak JD, Bellantone R, Raffaelli M, Fahey 3rd T, Vriens MR, et al. Hemodynamic instability during surgery for pheochromocytoma: comparing the transperitoneal and retroperitoneal approach in a multicenter analysis of 341 patients. Surgery 2018163 176-182. (https://doi.org/10.1016/j.surg.2017.05.029)

20 Kinney MA, Narr BJ \& Warner MA. Perioperative management of pheochromocytoma. Journal of Cardiothoracic and Vascular Anesthesia 200216 359-369. (https://doi.org/10.1053/jcan.2002.124150)

21 Kiernan CM, Du L, Chen X, Broome JT, Shi C, Peters MF \& Solorzano CC. Predictors of hemodynamic instability during surgery for pheochromocytoma. Annals of Surgical Oncology 201421 3865-3871. (https://doi.org/10.1245/s10434-014-3847-7)

Received in final form 12 March 2020

Accepted 17 March 2020

Accepted Manuscript published online 17 March 2020
This work is licensed under a Creative Commons Attribution-NonCommercial 4.0 International License. ded from Bioscientifica.com at 04/26/2023 10:19:40AM 